\title{
ON ALGEBRAIC CLOSURES
}

\author{
R. RAPHAEL
}

Abstract

This is a description of some different approaches which have been taken to the problem of generalizing the algebraic closure of a field. Work surveyed is by Enochs and Hochster (commutative algetra), Raphacl (categories and rings of quotients), Borho (the polynomial approach), and Carson (logic).

Laler work and applications are given.

"le was a man, take him for all in ahl, I shall not look upon his like again"... Harnlet.

\section{Introduction}

The study of field theory, of algebraic extensions, and algebraic closures for fields is fundamertal in algebra. These notions have many applications in mathematics, and have their own intrinsic intercst.

It is natural to consider the question of their generalization to other algebraic systems. In the late sixties and early seventies work was undertaken that led to such generalizations in commutative algebra. It is the purpose of this article to present the different approaches that were followed, to comment upon their methods, and to indicate the work which has been done more recently. The question of algebraicity was the topic of my last mathematical discussion with Pere Menal.

Thronghout this article all tings will be assuned to have an identity, and subrings are assumed to have the same identity element as their overrings. Rings will usually be commutative. A commutative ring is called semiprime (or reduced) if it has no nilpotents other than 0 . 


\section{The various approaches}

(a) Totally integrally closed rings. The work of Enochs.

An extension $S$ of a commutative ring is callect integral if every element of $S$ satisfies a monic equation with coefficients from $R$. Much is known about integral extensions, particularly about the relative behaviour of prime ideals under integral extension [22].

Relative to integral extensions Enoclis made the following formal definition:

Definition 1 [12] (Enochs). A ring $R$ is called totally integrally closed if for any ring homomorphism $\alpha: S \rightarrow R$ and any integral extension $T$ of $S$, there is a homomorphism $T \longrightarrow R$ extending $\alpha$.

Diagrammatically one has

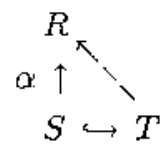

This is recognized as the diagram for injectivity, so totally integrally closed rings are injectives in an appropriate setting, -they are the injectives in the category of commutative rings with the restriction that the horizontal monomorphism in the diagram for injectivity be integral. (In the category of commutative rings as a whole the only injective object is the zero ring [19]). Enochs studied these rings and showed that the totally integrally closed fields were exactly the algebraically closed ones. His main results were:

Theorem 2 [12]. A commutative ring is a subring of a totally integrally closed ring if and only if it is reduced (semiprime).

Theorem 3 [12]. If $A$ is a commutative semiprime ring there is a totally integrally closed integral extension $A^{\prime}$ of $A$ which is also a tight extersion of $A$. If $A^{\prime \prime}$ is any other such extersion of $A$, then any $A$ homomorphism $A^{\prime}-\rightarrow A^{\prime \prime}$ is an isomorphism.

Enochs used the techniques of comnutative algebra, including localization and the properties of integral cxtensions. The results are derived directly from the definition of totally integrally closed rings, which are investigated as objects of interest in their own right. Theorem 3 is dcscribed as an analogue to the injective hull of a module-and its significance as a theorem on algebraic closure is clear. He notes that a rnonic polynomial $f$ has a spliting ring -a. tight integral extension generated 
by roots of $f$-with universal properties. Other interesting remarks are offered, and a question about the preservation of the property "totally integrally closed" under localization is posed.

(b) The work of Hochster. Totally integrally closed rings, and applications to rings of continuous functions.

Hochster wrote two articles on this topic shortly after Enochs' work was completed. "Totally integrally closed rings and extremal spaces" [15] has broad results and is the more algebraic in flavour. It gives several characterizations of totally integrally closed rings (Proposition 1), shows that their spectra must be extremal spaces, and discusses the factoring of monics into linear factors. It proves the interesting result that distinct minimal primes in a totally integrally closed ring must be comaximal, and that the relative topology on the mininal primes must be that of an extremally disconnceted Boolean space. Hochster discusses von Nennam regularity, and answers (in the negative) Enochs' question about localizations of totally integrally closed rings. The methods are those of commutative algebru with mach analysis of the spectrum.

The second article focuses on one particular kind of commutative semiprine ring those of the form $C(X)$, the ring of all continuous realvalued functions defined on a (completely regular) topological space $X$. $\left[C^{*}(X)\right.$ is the subring of functions in $C(X)$ which are bounded]. These rings are the subject of an entire text [13]. Much is known about their algebraic properties, in particular about the structure of their prime and maximal ideals.

Hochster considered and solved the question - "when is a ring of continuous functions totally integrally closed?". To understand his result we first need the following notion:-a topological space $X$ is called extremally disconnected if the closure of each open set is open. Discrete spaces are extremally disconnected, but so are mary others. The characterization is as follows:

Theorem 4 (Hochster). [14] The following are equivalent for a completely regular topological space $X$ :

(a) $X$ is extremally disconnected,

(b) $C(X)[i]$ is tolally integrally closed,

(c) $C^{*}(X)[i]$ is tolally integrally closed.

Hochster's methods in this article use both topology and algebra and are different from those in Enochs' article. His work extended that of Enochs, gave interesting characterizations of totally integrally closed rings: solved the localization problem, and characterized a specific topological casc. 
(c) Algebraic closures. The work of Raphael: Categories and rings of quotients.

Raphael, unaware of the work by Enochs, was attempting to generalize the construction of the algebraic closure for fields. As a student of Lambek, his methods were more categorical. In any category a monomorphism $\alpha: A-\rightarrow B$ is called essential if any morphism $\beta: B-\rightarrow C$ must be a monomorphism whenever the composition $\alpha \beta: A--\rightarrow C$ is a monomorphism. The best-known instance occurs in the category of right modules over a fixed ring. Fnochs used this notion in commutative rings and called such extensions "tight". Lambek pointed out that in the category of commutative rings an essential extension of a field $F$ is precisely a field extension, and suggested using the following which is easily checked:

Lemma 5. Let $F$ be a field and let $K$ be an extension of $F$. Then the following are equivalent:

(i) $K$ is algebraic over $F$,

(ii) every ring between $K$ and $F$ is a feld,

(iii) every ring between $K$ and $F$ is an essential extension of $F$ in the category of commutative rings.

Lambek also underlined the irnportance of regular rings as the correct generalization of fields to be considered. Raphacl made the following definition, -(he later added the adjective "weak").

Definition 6. Let $R$ be a conmutative semiprime ring, and let $S$ be an extension of $R . S$ is a weak-algebraic extension of $R$, if every between ring of $R$ and $S$ is an essential exterision of $R$, -i.e. every non-zero ideal in every between ring has non-zero contraction to $R$.

The following is immediate:

Lemma 7. Let $S$ be an extension of a commutative semiprime ring $R$. Then the following are equivalent:

(i) $S$ is weak-algebraic over $R$,

(ii) every non-zero element of $S$ satisfies a polynomial equation over $R$. whose constant term is non-zero.

This definition had the virtue of being "categorical" but suffered from two drawbacks. The minor one was that one could not prove transitivity for such extersions. The more important one was that it made the embedding of an integral domain into its field of quotients algebraic: and this seemed too general and untratural. Ultimately, the following definition was made. 
Definition 8. $S$ is an algebraic extension of $R$. if $S$ is essential over $R$, and if $S$ is integral over $R$. One has at once:

Lemma 9. Let $S$ be an extension of $R$. The following are equivalent:

(i) $S$ is algebraic over $R$,

(ii) every non-zero element of $S$ satisfies a monic polynomial over $R$ whose constant term is non-zero.

This notion had the advaritage of access to the existing results on integral extensions, particularly the theorens concerning the behaviour of prime ideals. Transilivity was immediate. The final result read:

Theorem 10. Let $R$ be a semiprime ring. Then $R$ has an algebraic extension which is algebraically closed (called an algebraic closure of $R$ ). An algebraic closure contains a copy over $R$ of any algebraic extension of $R$. Any tus algebraic closures of $R$ are isomorphic over $R$.

With this result it was possible to return to weak algebraic extensions and get the analagous result for them by using the theory of rings of quoticnts. The weak-algebraic closure is the conplete ring of quoticnts of the algebraic closure. Only then (using abstract nonsense) did the transitivity of weak-alyebratic extensions follow. No direct proof for the transitivity has yet been givon.

In summary, Raphael's work was rooted in the ideas of categories, rings of quotients, and the study of regular rings. Baer rings also arosea commutative ring is Baer if the annililator of every subset is a principal ideal generated by an idernpotent. A regular ring is algebraically closed preciscly when it is Baer and all of its factor fields are algebraically closed. The algebraic closure of a semiprime ring $R$, can be obtained by passing to its complete ring of quotients $Q(R)$, known to be regular, taking the algebraic closure of $Q(R), \Omega(Q(R))$ and finally taking the integral closure of $\mathrm{R}$ in $\Omega(Q(R))$. In the general serriprime case, an algebraically closed ring must also be Baer- (this was Hochster's demand that the spectrum be extremal) because one could easily get an algebraic extension by taking the integral closure in the complete ring of quotients which is Bater.

\section{(d) The work of Borho. The polynomial approach.}

Borho's work was subsequent; to that of Enochs, Hochster, and Raphael. His study was "a contribution to the question: Is there any reasonable analogine for the algebraic closure of a ficld,... for an arbitrary commutative ring?" Together with Weber [4] he gave a proof of 
Enochs' Theorem 2 above. His thesis approaches the problem of algebraic closure by studying essential extensions, extensions which acjoin roots of polynomials, and extensions by idempotents. If $f$ is a monic polynomial over $R$, then an $f$-extension is a ring generated by $R$ and roots of $f$. If $F$ is a set of monics over $R$, then an $F$-extension is one generated by $R$ and roots of elements of $F$. An $F$-splitting extension is an $F$-extension in which every element of $F$ can be written as a product, of linear factors. An essential $F$-splitting extension always exists for a given $R$, and $F, R$ is called $F$-saturated if it has no proper essential $F$-extensions. Borho determined $[3,4.2 j$ when an $F$-saturated extension exists. It must exist for ary $F$ if $R$ is semiprime. A monic polynomial $f$ is called "saturable" if the number of zeros it can have in an essential extension of $R$ is bounded. All monics are saturable in the semiprine case, but in general they can fail to be. A "saturable closure" exists for all commutative rings. It coincides with Enochs' total integral closure in the semiprime case. There are non-semiprime cases, for cxample, Noctherian irreducible rings, where uniqueness holds, but in general it fails. The saturable closure can be viewed as the result of two steps -the first by adjoining idempotents, and the second by taking a splitting extension. In the semiprime Baer case the first step is unnecessary.

Borho's work gave an independent, approach to the subject which reestablished the semiprime results, and analyzed the pathology of the general commutative casc.

\section{(e) Carson-a logical approach.}

Carson studied algebraically closed commutative regular rings from a logical and sheaf-theoretic point of view. Such in study is suggested by the fact that commutative regular rings form an equational category -to the usual axioms for commutative rings one adds the demand that the unique quasi-inverse exist for each element. Carson's terrninology is different, however, and consequently, his results are as well. A commutative regular ring is algebraically closed if it has a root for each of its monic polynomials. A sheaf of fields is algebraically closed if all the stialks are algebraically closed fields. A shcaf $K$ of fields over a Boolean space $X$ is an algebraic closure of a sheal $k$ of fields over $X$, if $k$ is a subsheaf of $K_{\text {: }}$ and if for each $x \in X, K_{x}$ is the algebraic closure of $k_{x}$. The algebraic closure of a sheaf necd neither exist nor be unique. [7, p. 1038] However when the ring is Baer [7, Corollary 1.6$]$ an algebraic closure will be unique up to isomorphism, and under certain general conditions two algebraically closed rings will be clementarily equivalent. [7, Theorem $2.1]$

The characterization of algebraically closed regular rings is the theme 
of Carson's work.

\section{Later developments}

(a) The non-commutative question. At some point the question was bound to arise as to whether a non-commutative generalization was possible. No very satisfactory solution bas been found. One of the obstacles is the need for a well behaved notion of integrality. Raphacl decided to use that due to Atterton [1]; -let $R$ be a subring of a ring $S$. $s \in S$ is integral over $R$, if there is a finitely generated $R$-rnodule $M$ with generators from $Z(S)$, the centre of $S$ : such that $1 \in M$ and $s M \subseteq M$. $S$ is called integral over $R$ if all elements of $S$ are integral over $R$. A ring embedding $R--\rightarrow S$ is algebraic if it is integral and essential (in the category of rings). It turns out that for rings whose Pierce stalks are nice enough, an algebraic closure exists. [20, Theorem 13]. The least technical result is perhaps

Theorem 11. Let R be regular, right self-injective, directly finite, and suppose that $Q$ (the rational field) lies in $Z(R)$. Then $R$ is algebraically closed iff $Z(R)$ is.

The conditions required in this theorem give some indication of the limitations of the results. As a byproduct, the non-commutative work gave a new commutative result: [20, Corollary 23 ] If $R$ is commutative regular self-injective, and $R[a]$ is algebraic over $R$, then $R[a]$ is also selfinjective.

(b). Macoosh and Raplael [17] investigated the notion "totally integrally closed" for Azumaya algebrus, and showed that an Azmmaya algebra is totally integrally closed exactly when its centre is. Enochs' theorem on algebraic closures generalizes to Azumaya algebras over commutative semiprime rings. Difficulties are encountered when one moves to more general non-commutative rings.

In a separate article [16] they gave a short proof of (Hochster's) Theoren 4 using techniques from the theory of rings of quotients of rings of functions.

\section{(c) The hereditary question.}

Suppose that $R$ is a commulative ring, and that $B(R)$ is its set of idempotents. $B(R)$ can be made into a ring by keeping the sume nultiplication as in $R$, but defining the addition by $e \oplus f=e+f-2 e f$. $B(R)$ is called the Boolean ring of idempotents of $R$, and is useful for 
the Pierce sheaf representation of $R$. A commutative ring $R$ is called hereditary if its ideals are projective as $R$-modules. The question arose as to whether the hereditary property was preserved under finite algebraic extension. In general the answer is no -the simplest example being $Z[\sqrt{3}]$ or any other non-Dedekind finite extension of $Z$. For the regular case, however, one can make use of Bergmann's work which says:

Theorem 12 (Bergman). [2, Corollary 4.6, Lemma 1.1] The following are equivalent for a commutative regular ring $R$ :

(i) $R$ is hereditary,

(ii) $B(R)$ is hereditary,

(iii) every ideal of $R$ is generated by an orthogonal set of idempotents.

By working with idempotents one gets the desired rcsult for the regular hereditary case:

Theorem 13. [11]. Let $R$ be a commutative hereditary regular ring, and let $S=R\left[a_{1}, a_{2}, \ldots, a_{n}\right]$ be an algebraic extension of $R$. Then $S$ is a hereditary regular ring.

\section{(d) The Galois question.}

With a solution of the problem of algebrajcity available, one would expect a discussion of separability and Galois theory. Fortunately, a relevant theory had already been developed. Let $R$ be a subring of $S$. Form $S \underset{R}{\otimes} S$ a ring which acts naturally on $S$.

$S$ is a separuble $R$ algebra [8] if $S$ is projective over $S \otimes \underset{R}{\otimes} S$. When $R$ and $S$ are fields this is equivalent to the demand that $S$ be a finite separable extension of $R$ in the usual sense.

$S$ is a Galois extension of $R$ if the following three conditions hold [8]:

(i) $R$ is the fixed ring of a finite group $G$ of automorphisms acting on $S$;

(ii) $S$ is a separable $R$ algebra,

(iii) for each non-zero idempotent $e \in S$, and each pair $\sigma \neq \tau$ in $G$, therc exists $x \in S$ with $\sigma(x) e \neq \tau(x) e$. It follows that $S$ is finitely generated and projective as a module over $R$. If 0 and 1 are the only idempotents of $S$, then condition (iii) can be ignored. Chase, Harrison, and Rosenberg [8, p. 10, Theorem 2.3] showed that, if $S$ is a Galois exterision of $R$, with Galois group $G$, then there is a Galois-type theorem exhibiting a $1-1$ correspondence between the subgroups of $G$ and certain separable $R$-subalgebras of $S$. 
Villamayor and Zelinsky [21] were interested in the case where $S$ can have an arbitrary number of idcmpotents. [21] $S$ is a weak Galois extersion of $R$ if $S$ is a separable $R$ algobra, if $S$ is a finitely generated projective $R$-module, and if $R$ is the fixed ring of a finite group of automorphisms of $S$.

If $R$ has no idempotents different from 0 and 1 then a weak Galois extension $S$ is Galois. In general, when $S$ is weakly Galois over $R$, there exists a finite set of orthogonal idempotents $\left\{e_{1}, \ldots, c_{n}\right\}$ in $R$ with $\sum e_{i}=$ 1, such that $S e_{i}$ is Galois over $R e_{i}$ for each $i$. As well, a Galois-type theorem pairs the separable $R$-subalgebras of $S$ with certain subgroups of the group of automorphisnis of $S$ over $R$ [21. Theorem 3.8].

A last notion is due to DeMeyer $[9] . S$ is a normal separable extension of $R$ if $S$ is separable over $R$, if $S$ is projective over $R$, and if $R$ is the fixed ring of the group of $R$-automorphisms of $S$.

Should $R$ be the fixed ring of a finite group of $R$-automorphisms of $S$, then $S$ is weak-Galois over $R$, but this can fail to occur. One has strict implications from Galois to weak Galois extensions and from weak Galois to normal separable extensions.

The relevance of these notions to algebraic extensions was studied by Desrochers and Raphael $[\mathbf{1 0}]$. The results require assumptions which prevent the extensions from gaining idempotents. The main results are:

Theorem 14. $[10,4.1]$ let $R$ be regular and suppose that $B(R)=$ $B(R[a])$, where $R$ is semiprime and $R[a]$ is an algebraic extension of $R$. Then $R[a]$ is separable as an $R$-algebra iff for every $t, \in X(R)$, the field extension $R[a]_{x} / R_{s}$ is classically separable.

Theorem 15 (Primitive element). Let $R$ be regular and let $S=$ $R\left[a_{1}, \ldots, a_{n_{t}}\right]$ be an algebraic extension of $R$ such that $S$ is separable over $R$ and $B(S)=B(R)$. Then $S=R|b|$ for some $b \in S$.

Theorem 16. Let $R$ be regular, and let $R[a]$ be an algebraic extension of $R$ that is $R$-projective, R-separable, and such that $B(R)=$ $B(R[a])$. Then $R[a]$ is weakly Galois over $R$ iff for all $x \in X(R)$, the field extension $R \cdot[a]_{x} / R_{x}$ is normal.

Theorem 17. Let $R$ be regular (Baer) integrally closed in its complete ring of quotients, and let $R[a]$ be an algebraic estension of $R$. Let $p(t)$ be a monic of minimal degree over $R$ satisfied by $a$, and let $S=R\left[\sigma_{1}, \ldots, \sigma_{n}\right]$ be the splatting ring of $p(t)$ in an algebraic closure of $R[a]$. Then $S$ is weakly Galois over $R$. if $S$ is a seporable R-algebra, or equivalently; if for every $x \in X(R), S_{x}$ is separable over $R_{\%}$. 


\section{References}

1. T. W. ATTERTON, Definitions of integral elements and quotient rings over non-commutative rings with identity; J. Austr. Math. Soc. 12 (1972), 433-446.

2. G. M. BERGMAN, Hereditary commutative rings and centres of hercditary rings, Proc. London Math. Soc. 3, 23 (1971), 213-236.

3. W. BoRHo, Wesentliche ganze Erweiterungen konnutativer Ringe, Dissertation, Hamburg (1973).

4. W. Borho AND V. WeBer, Zur Existenz total ganz abgeschlossener Ringerweiterungen, Malh. Z. 121 (1971), 109-111.

5. N. BourbaK1, "Elements de mathematique, Algebre commutative," Fasc 27, chapitre 1: Modules plats; chapitre 2: Localisation, Hermann, Paris, 1959.

6. A. B. CARSON, Representation of semisimple algebraic algebras, $J$. of Algebra 24 (1973), 245-257.

7. A. B. Carson, Algebraically closed regular rings, Cam. J. Math. 26 (1974), 1036-1049.

8. S. Chase, D. Harrison and A. Rosenterg, Galois theory and cohomology of commutative rings, Mem. Amer. Math. Soc. 52 (1965).

9. F. DeMEYER, On the automorphisms of separable algebras 2, Pac. J. Math 32 (1970), 621-631.

10. M. C. Desrociers and R. M. Raphael, Galois Theory and algebraic extensions of commutalive semiprime rings, Comm. in Alg. 18, 3 (1990), 927-946.

11. M. C. Desrochers and R. Raphase, On algebraic cxtensions of hereditary regular rings; (Submitted).

12. E. ENochs, Totally integrally closed rings, Proc. Amer. Math. Soc. 19 (1968), 701-706.

13. L. Gillman and M. Jerison, "Rings of Continuous Functions," Van Nostrand, 1960.

14. M. HOCHSTER, Rings of continuons functions and totally integrally closed rings, Proc. Amer. Math. Soc. 25 (1970), 439-442.

15. M. Hochster, Totally integrally closed rings and extremal spaces, Pac. J. Math. 32 (1970), 767-779.

16. R. MACOOSH AND R. RAPHALL, Totally integrally closed rings of continuous functions and rings of quotients, C.R. Malh. Rep. Acad. Sci. Canada 11, 3 (1989). 
17. R. Macoosh and R. RAPHAEL, Totally integrally closed Azumaya algebras, Can. Math. Bull. 33, 4 (1990), 398-403.

18. R. RAPHAEL, Algebraic extensions of commutative regular rings, Can. J. Math. 23 (1970), 1133-1155.

19. R. RAPHAEL, Injective rings, Comm. in Algebra 1 (1974), 403-414.

20. R. RAPIABL, Algebraic closures for some non-conmutative rings, Comm. in Alg. 17, 7 (1989), 1687-1708.

21. O. E. VILLAMAYOR AND D. ZELINSKI, Galois theory with infinitely many idempotents, Nagoya Math. J. 35 (1969), 83-98.

22. O. ZARISKI AND P. SAMUEL, "Commutative Algebra," Vol. 1, Springer-Verlag, New York, Heidelberg, Berlin, 1958.

Mathematics Dcpartment

Concordia University

Montrcal

CANADA

Rebut el 8 d'Octubre de 1991 